Please do not remove this page

RMIT

UNIVERSITY

\title{
Active engagement with stigmatised communities through digital ethnography
}

Barratt, Monica; Maddox, Alexia

https://researchrepository.rmit.edu.au/esploro/outputs/9921899907901341/filesAndLinks?institution=61RMIT_INST\&index=null

Barratt, M., \& Maddox, A. (2016). Active engagement with stigmatised communities through digital ethnography. Qualitative Research, 16(6), 701-719. https://doi.org/10.1177/1468794116648766 Document Version: Accepted Manuscript

Published Version: https://doi.org/10.1177/1468794116648766

Repository homepage: https://researchrepository.rmit.edu.au (C) 2016 The Author(s)

Downloaded On 2023/04/26 14:14:47 +1000 
Barratt, M. J., \& Maddox, A. (Published online before print 22/5/16). Active engagement with stigmatised communities through digital ethnography. Qualitative Research

Link to published version:

http://qrj.sagepub.com/content/early/2016/05/20/1468794116648766.abstract

Active engagement with stigmatised communities through digital ethnography

Monica J Barratt

- Drug Policy Modelling Program, National Drug and Alcohol Research Centre, UNSW Australia, Australia

- National Drug Research Institute, Faculty of Health Sciences, Curtin University, Australia

- Burnet Institute, Centre for Population Health, Australia

Alexia Maddox

- National Drug Research Institute, Faculty of Health Sciences, Curtin University, Australia

- Library Research Services, Deakin University, Australia

Corresponding author:

Monica J. Barratt

Drug Policy Modelling Program

National Drug and Alcohol Research Centre

UNSW Australia, Sydney NSW 2052, Australia

Phone: +61407778938

Email: m.barratt@unsw.edu.au

Word count (body of text $)=7546$ 
Title: Active engagement with stigmatised communities through digital ethnography

Keywords [up to 10]: community-based research, digital ethnography, ethics, workflow, illicit activities, stigmatised populations, drug use communities, digital trace analyses, Big Data

\begin{abstract}
Conducting research in the rapidly evolving fields constituting the digital social sciences raises challenging ethical and technical issues, especially when the subject matter includes activities of stigmatised populations. Our study of a dark-web druguse community provides a case example of 'how to' conduct studies in digital environments where sensitive and illicit activities are discussed. In this paper we present the workflow from our digital ethnography and consider the consequences of particular choices of action upon knowledge production. Key considerations that our workflow responded to include adapting to volatile field-sites, researcher safety in digital environments, data security and encryption, and ethical-legal challenges. We anticipate that this workflow may assist other researchers to emulate, test and adapt our approach to the diverse range of illicit studies online. In this paper we argue that active engagement with stigmatised communities through multi-sited digital ethnography can complement and augment the findings of digital trace analyses.
\end{abstract}

Abstract word count [150 word limit]: 150 words 


\section{Introduction}

The study of stigmatised or marginalised communities comes with a recognised set of challenges. Access is problematic, it can be more difficult to conceptualise the issues, and the relationship between researcher and participant is likely to be "hedged about with mistrust, concealment and dissimulation" (Lee, 1993: 2). Such challenges stem from the participants' legitimate concerns about disclosing information to researchers that, if known more widely, could have serious negative repercussions for their lives, including social isolation, termination of employment, and criminal penalties. It is much harder for researchers to get into contact with members of stigmatised communities and to build the necessary rapport required to engage research participants. Ethnography is well-suited to the study of marginalised or hidden communities, and there are many examples of classic ethnographies that achieve rich description and understanding of people's experiences that would have otherwise been hidden from view (as reviewed by Adler, 1990).

More recently, the use of globally networked, digital technologies has increased to the point of becoming an unremarkable part of everyday life for most citizens in the global north (with the global south rapidly increasing its internet penetration also, Pew Research Center, 2014). Early sociological inquiry into how internet technologies were used demonstrated that the internet was particularly attractive to stigmatised communities (McKenna and Bargh, 1998; King, 1994). People who identified as nonnormative or who behaved in non-normative ways were able to connect with other like-minded people without having to reveal the non-normative identity or behaviour to people in their everyday lives. Through this digitally mediated interaction, such people could share information, receive and provide support, and spend time maintaining a sense of belonging to the group (Tanis, 2008).

The attractiveness of the internet to stigmatised groups did not go unnoticed by social researchers. In our area of research with people who use illicit drugs, researchers were quick to utilise the internet to recruit the target population as research participants (Barratt and Lenton, 2010) and to host anonymous web surveys (Miller and Sønderlund, 2010). There are also some examples of qualitative interactive studies of this population using digital technologies, but these are less common (Coomber, 2011). But another type of study became possible through the increased embeddedness of digital technologies in everyday life: analyses of digital traces (Ackland, 2013) or native digital data objects (Lupton, 2015). These are data produced through the interactions between people and digital technologies. Unlike in-person interactions, digital interactions leave traces that can be easily copied into forms that can be transmitted quickly and globally (Ess, 2009). For stigmatised populations, the characteristics of these data have opened up new horizons for unobtrusive observation in research practice (Décary-Hétu and Aldridge, 2015).

This fundamental change in data generated through social interaction and the very operation of digital technologies has fuelled excitement about the capacity of 'Big Data' to change how we live, alongside caution (Ekbia et al., 2015). Claims about large-scale quantitative analyses of digital traces (or webometrics) being more 
'complete' or less 'biased' than surveys or interviews are premised on assumptions that native digital data objects are produced, stored and analysed 'objectively', yet researchers must choose what to select and what to store and often must rely on 'black box' media analysis tools, built by and for corporate interests (Bruns, 2013). Furthermore, the meaning of the data may be lost or misinterpreted when taken out of the social and cultural context within which it was produced (boyd and Crawford, 2012; Lupton, 2015). This critique of Big Data emerging within the literature, alongside a discussion of its possibilities, suggests that there are limits to what researchers can expect from these new digital artefacts of social behaviour, both in terms of interpretation and representativeness.

Within this context, we argue that interactive digital ethnography can act as a contextual counter-balance to the increasing use of unobtrusive analyses of digital traces in the study of stigmatised populations online. Ethnographers seek to explain practices that appear 'irrational' from one perspective by providing rich description of the cultural logics of the researched group, to whom the practice makes sense (Northcote and Moore, 2010). In this way, ethnographers translate or interpret the cultural logics of one culture into another (Agar, 2011). Ethnographers learn these cultural logics by engagement in the field, through interaction with members of the cultures the ethnographer seeks to understand. The use of ethnographic methods in digital spaces - digital, virtual, online or internet ethnography (Hine, 2008; Pink et al., 2015) — is not fundamentally different from traditional ethnography as described above, although the digital environment requires adaptation to account for the nature of digital information (traceable yet ephemeral, easily copied, transmitted quickly and globally) (Lupton, 2015). In this paper, we demonstrate the value of the digital ethnographical approach toward the study of stigmatised communities where we maintained an active presence within the digital field-sites. This fieldwork sensitised us to better contextualise the information we produced through observation and interview.

Others have written about the ethical and methodological issues relevant to the conduct of digitally mediated research (e.g., Whiteman, 2012; Ess, 2009; McKee and Porter, 2009). While it is not the goal of this paper to broadly review digital research ethics, we wish to add to these discussions and situate them within the current context of the internet becoming increasingly identified or authenticated, leaving less and less room for the anonymity required to discuss stigmatised activities freely. Government and corporate internet content filtering is becoming more commonplace (Koumartzis and Veglis, 2011), and the increased use of proprietary websites and mobile devices also places restrictions on the freedom of content available to users of those sites and devices (Barratt et al., 2013). In one example, Facebook has a username policy that requires users sign up using their 'real name'. Facebook's business model relies on the convergence of data for targeting advertising, therefore it is not in Facebook's interest for people to operate accounts with alternate un-linked identities. Although some Facebook users successfully subvert the real name policy (Raynes-Goldie, 2010), the overall trend is towards an identified and authenticated internet. We have previously argued that this trend has pushed people who seek anonymity towards private 
networks and the 'dark net' or 'dark web' (Barratt et al., 2013). The dark net is a part of the internet that is not indexed by conventional search engines and is only accessed by specialized browsers: for example, onion sites must be accessed using the Tor (The Onion Router) browser, and this software routes internet traffic through a series of relays to make it much more difficult to track the identities and locations of network users (Dingledine et al., 2004). A unique contribution of our article, then, is to describe and justify the decisions made within our research within the context of an identified internet and an alternate anonymous internet, the 'dark net', with the aim to assist other researchers when planning their work in similar domains.

In this paper, we demonstrate the practice, rigour and workflow of an ethnographic approach to the study of a stigmatized community online. In doing so, we take into account the increasing shift of these populations into the dark net and document the related issues and considerations that engaging participants in this domain of the internet raised for our ethnographic workflow. We argue that whilst the issues we consider here of field-site volatility, researcher safety and ethical-legal issues are likely to apply for those conducting unobtrusive observations through digital trace data analysis, their imprint upon the data may not hold the coherent and meaningful narrative that became apparent through our active and engaged participatory approach. From the contextual foundation that this research brings to the study of stigmatized populations online, and more particularly in the anonymised spaces of the dark net, we anticipate that researchers will be able to adopt, develop and appropriate the workflow and learnings that we present here. In addition to this, we anticipate that further unobtrusive analysis of digital trace data in this environment will be able to draw on the findings of this study in order to contextualize and interpret the social and behavioural patterns observed.

\section{The case study: 'Silk Road'}

Our project was conducted with people who purchased illicit drugs from the online anonymous market or cryptomarket, Silk Road, whom we engaged to assist in our understanding of the social impacts of cryptomarkets upon drug-use communities (Maddox et al., 2016; Barratt et al., 2016). Cryptomarkets are a recent socio-technical innovation, aligned with an ethos of information liberation, that provide autonomous market activity outside of the centralized control of governments. To facilitate exchange they use cryptocurrencies, such as Bitcoin, which are decentralized, peer-topeer digital cash systems that operate through encryption techniques and independently of the banking system. Cryptomarkets leverage this alternative financial system to provide unregulated and decentralized online marketplaces for the exchange of goods and services, in a manner similar to eBay (Barratt, 2012b; Barratt et al., 2014). They are hosted in the dark net, as described above.

The study of cryptomarkets is a nascent field, mainly characterized by an unobtrusive observational approach, usually combined with quantitative analytic approaches (e.g., Aldridge and Décary-Hétu, 2014; van Buskirk et al., 2014; Soska and Christin, 2015). An exception is van Hout and Bingham's work (2013b; 2013a; 2014) which employed 
ethnographic methodologies including observations, engaging in discussions, and conducting qualitative interviews within the dark net environment. Our study built on van Hout and Bingham's pioneering ethnographic study of the original cryptomarket, Silk Road, which offered the first characterisations of the development of a drug use community within its anonymous and encrypted confines.

\section{Developing an ethnographic workflow in the dark net}

We conducted digital ethnographic work through 2012-2014 after the study was approved by the Curtin Human Research Ethics Committee (HR 26/2012). ${ }^{1}$ Our interactive fieldwork period (September 2013 to June 2014) spanned the period just before, during and after the seizure of the marketplace by the U.S. Federal Bureau of Investigation on 2 October 2013. As far as we know this period was the first significant disruption caused by law enforcement activity experienced by Silk Road users.

The ethnographic workflow we developed in this study for engaging the Silk Road community contained three stages. Stage 1 involved our preparation for entering the field; Stage 2 included field entry, engagement and exit; and Stage 3 involved knowledge production and dissemination.

\section{Preparation}

Stage 1 was characterised by the development, design and preparation of the research. During this period we initially observed the population and considered the technical, ethical-legal and social sensitivities; with a particular emphasis upon handling sensitive information and preserving participant anonymity through approaches to data management and the use of appropriate technologies for engagement.

Conducting digital ethnography in the dark net requires a strong working knowledge of the technical practices that are used to maintain anonymous communications. This approach responds to an ethos of anonymity held by techno-elite communities, in addition to the range of illicit practices associated with the retail of drugs in the unregulated and global reach of cryptomarkets. Because of these considerations for the conduct of this study, preserving participant anonymity became the foundation of the data collection process. Establishing these practices required a long project lead-in time to develop appropriate technical protocols and skills: including the encryption of email communication, use of encrypted browsers (e.g. Tor) and anonymising operating systems (e.g. Tails), selecting an appropriate encrypted online chat or instant messaging (IM) platform, and appropriate data management practices.

\footnotetext{
${ }^{1}$ The dynamic nature of this project and our responses necessitated multiple ethics amendments to our committee.
} 
Through community and expert consultation, we established that it was possible to conduct fully anonymous interviewing with participants if they engaged by the Pidgin IM program that comes preinstalled within the Tails operating system. Both of these programs are freely available and maintained as open source software. The Pidgin IM program facilitate online chats which occur synchronously (in real time). Encryption can be enabled within this program, and when the participant uses it within the Tails operating system, the interviewer cannot establish the identity of the participant by commonly used technical approaches such as retrieving their IP (Internet Protocol) address, a string of numbers which can potentially identify individual internet users (Rankin, 2014). While this process appeared to us to be ideal because it meant we could guarantee to participants that it would be impossible for us to identify them, setting up Tails required a high level of technical expertise. A more accessible alternative was proposed where participants set up instant messaging with the OTR plugin (Off The Record, an encryption plug-in, see Borisov et al., 2004) and a disposable account to conduct the interviews, but did not use the Tails operating system. While it was technically possible for us to access the participant's IP address through this alternative approach, many participants used other approaches that would not reveal identifying IP addresses to us, such as using public WIFI services or IP anonymizers. At no point did we attempt to access this information while conducting the interviews. While we did test the interview exchange through the Tails platform, it was not appropriate for us to use Tails during the interview, because that would make it impossible for the participant to verify our identity via matching our public key, hosted on the university website, with our fingerprint used at the start of the interview. The use of this process of verification was particularly successful at establishing that we were whom we claimed to be through technologies of trust rather than relying on the development of social trust.

Further technical aspects of our work during Stage 1 were the preparations we made to reduce our vulnerability to cyber attack and reduce the risks of data breach through third party access. Prior to community engagement, we realised that we would be vulnerable to identity theft and other cyber-attacks by publishing our real names in the dark net, so we assessed our digital security before entering this field by ensuring use of multiple unique and secure passwords on important accounts, and ensuring our computers had anti-virus protection. This technical level of researcher protection may be likened to the digital equivalent of preparing for both ambient and situational risks of in-person fieldwork encounters (Lee, 1994). During the design phase of Stage 1, we also considered the rationale for the use of overt methods of engagement, rather than the covert methods more commonly used with this population, with the aim of establishing community contribution and receptivity to the research. The insights generated during this process of the development of technical capacity and cultural practices of the community raised additional ethical considerations including researcher safety and the apparent need for the transparency of researcher identities and actions in the online environment (see later in this article).

\section{Interactive fieldwork}

Stage 2 covered the period of entering the field, engaging the research population and 
exiting the field. During the period of entering the field, we focused on the development of a credible researcher identity within the community and monitoring community dynamics in response to our presence. Entry into cryptomarkets is simple for anyone capable of downloading and running the freely available Tor software; however, the methodological/ethical decisions upon access are more complex. As argued by Knox (2001), beyond access, the first requirement for researchers in undertaking fieldwork is to establish their legitimacy within the community. A key component of this process, he argues, is demonstrating to the community that the researchers will approach the study fairly or objectively. To achieve this, we were transparent on our position as researchers through our overt connection to the study website hosted by Curtin University that also contained an online plain language statement for the study, our public encryption keys and university email accounts (see also Barratt, 2012a). We also presented a consistent virtual presence through our digital activities, using our real names rather than pseudonyms across the clear web and dark net environments. This approach sought to allow for independent verification of our identities by the community and to establish the shared trust between researchers and participants through a clear statement of our affiliation and agenda in engaging with a drug use community (Crowley, 2007). Although we were not insiders to the community, we were not completely outsiders either. M.B., for example, could point to her longstanding voluntary role as administrator at Bluelight.org, a drug harm-reduction clear-web forum that was well regarded on Silk Road, and her research papers, blog posts and mainstream media contributions on the topic of Silk Road. We used this pre-existing digital presence to demonstrate our commitment to values, such as harm reduction, that we deemed likely to be shared by many community members.

In contrast to our approach, Gehl (2014), in his ethnography of a dark net social network, chose to remain pseudonymous, as he observed that it was not culturally appropriate to reveal real-world names in that environment. Our rationale in remaining identified was to allay participant concerns about our identity as researchers, given the reality of undercover agents operating in this space. However, operating as identified individuals in the dark net raised a further concern about our digital (and real-world) safety (see below). In hindsight, it could have been useful to operate dual identities, such that one remained the identified researcher, and the other could blend in more effectively through use of a pseudonym and without having to account for the research in online encounters. This technique was utilized by Paechter (2013), who maintained her pseudonymous account while conducting research using her identified researcher account (the connection between the two accounts was only known by the community's management group). While the two accounts afforded her greater access as an insider, ethical concerns arising from dual roles became harder to manage as material which was provided through interaction with her insider account could not be 'unknown' by her research identity. By engaging with the research population during the second stage of the research, initially the Silk Road marketplace and forums, and then through other channels the population became active, we worked to provoke a community-wide response through the development of a recruitment thread. An advantage to developing and maintaining a recruitment thread in community spaces 
online is that it provides a publically accessible and community archived transcript through which community members and researchers can evaluate proposed research endeavours based upon the conduct of previous ones. Van Hout and Bingham (2013a: 386) note in their first attempt to access the community: "recruitment of site users was hampered by negative and suspicious reactions by forum participants". Community responses to our call for participation in the dark net forum recruitment thread demonstrated a negative reception characterised by accusations of the researchers being "community outsiders". Within the context of an online community that has been active and sustained over time, the space for the internal moderation and regulation of community responses can be left open to establish whether there is a receptive space within the community to conduct the research (c.f., Williams, 2007). For example, in response to a particularly dismissive comment on what the research could achieve, one poster enacted a form of internal community regulation and control:

[...] I've never been into opiates myself, but I've known a lot of people who do and every single one of them made much more sense and was a lot smarter than you, you smug little prick. Now put on your pyjamas, brush your teeth and fuck off to bed. the adults are talking (the Hub forums, community member, 21.02.14)

As the above poster suggested in his final flourish, positive endorsement of the research in community responses to the recruitment thread positioned it as an opportunity for the community to present its point of view to the wider public through the non-judgemental lens of research practice. For example, in a subsequent post, one of the forum moderators endorsed the research, setting an influential tone for its reception within the community.

I think that research programmes like this are valuable to the long-term legitimacy of darknet markets and the darknet community as a whole. Thank you for conducting this research in an unbiased and respectful manner. (The Hub forums, moderator, 22.02.14)

The subsequent commentary suggested the efficacy of engaging gatekeepers, in this case forum moderators, as research advocates within the community. In direct messages with the researchers, it was also suggested by a forum moderator that there was greater receptivity within the community to the study proposed than was evident in the recruitment thread due to the fact that those with stronger opinions or aversions were more likely to engage publically. In addition to this, through the visibility of the community response to the recruitment posts, we identified an unresolved tension between Silk Road's bold public approach, as exemplified by the site administrator's State of the Road address (see the text excerpt below) and other community members who feared that increased publicity invited greater scrutiny by law enforcement, vilification by the media and increased infiltration of the community by lessknowledgeable outsiders.

Silk Road was never meant to be private and exclusive. It is meant to grow into a force to be reckoned with that can challenge the powers that be and at last give 
people the option to choose freedom over tyranny (Dread Pirate Roberts - Silk

Road Administrator, "State of the Road Address", Silk Road forums, 09.01.12).

During this stage of engaging the research population, the agentic nature of ethnography also became crucial to our capacity to deploy an exploratory, flexible and adaptive research practice. In our study of the cryptomarket Silk Road, we began with the aim of studying the community surrounding the Silk Road marketplace and forum, but quickly adapted our methodology to 'follow the people', as suggested by Marcus (1995), upon its seizure by law enforcement in October 2013. Once the site was seized, we suspended further active recruitment for interviewees, while continuing with passive monitoring of the broader dark net environment. Like McCormack et al. (2013), who planned online recruitment strategies but had to innovate quickly in the face of failure, this volatile environment required a change of approach. Upon modification of the research ethics supporting this project to take the digital migration of the community and retrospective approach into account, A.M. actively engaged with and recruited online interview participants via these channels as an identified researcher, and we were successful in interviewing 17 Silk Road buyers using encrypted instant messaging (IM). Consequently, the ethnographic approach proved powerful in allowing us to respond to unanticipated and emergent events within the community and be sensitive in our timing for rapport building and community engagement.

The practice of conducting qualitative research interviews using IM or other synchronous online chat modes has been described and assessed elsewhere (e.g., Ayling and Mewse, 2009; Davis et al., 2004; Barratt, 2012a). While most studies reporting synchronous online interviewing do not mention encryption, Ayling and Mewse (2009) embedded an IM program that was preinstalled with encryption into their project website and inviting interviewees to participate using a pseudonymous email account. In Barratt's previous work with drug users (2012a), encrypted IM was offered but was only attempted by 1 of 27 interviewees and then was dropped due to technical difficulties. In our study, we felt that it would not be ethically defensible to conduct these interviews without encryption given intense law enforcement interest in Silk Road (see later discussion of ethical-legal challenges). Using a tool similar to Ayling and Mewse would not be acceptable to this community due to their avoidance of acting within the clear net where they could be tracked (unless, perhaps, if it were hosted as a Tor hidden service). Therefore, we used the OTR tool which is an open source encryption plug-in, which also had strong support in the community. Through this tool we successfully enabled encryption for all 17 online interviews.

A core function of the interviews was member checking, or exercises to establish the veracity of research findings (Creswell and Miller, 2000). Throughout the interview, A.M. deployed techniques such as summarising her understandings of what had been discussed, using time checks to display sensitivity and value for the participants time, and using humour and language specific to IM interactions to develop rapport (see Barratt, 2012a). For many participants, the interview represented a confidential setting where they could share their experiences in a constructive and non-judgemental environment. We argue that this socially exceptional context added to their sense of 
engagement with the interviewer and was likely to have contributed to their value for the research in its aim to present the drug-user perspective. Given that the interviews were conducted in real-time and in a conversational manner, A.M. was able to raise events and observations as they were happening within the cryptomarket environments, such as Bitcoin heists and DDOS (Distributed Denial of Service) attacks, and share her perspective of these with participants. In doing so, we were able to confirm or reframe our understandings of these events.

\section{Knowledge production and dissemination}

Upon entering the final stage of the research, after completion of data collection, strategies relating to ethical conduct, technological affordances and community connection continued to inform collaboration surrounding the production, sharing and dissemination of research findings. At the latter stages of fieldwork and within the final stage of writing up, member checks were conducted with homologous communities of practice in-person. While in-person member checks may seem counter-intuitive to a digital ethnography of a marginalised community, they provided another medium through which to engage the community and for the researcher to embody the research practice. A.M. attended and presented interim findings at a local Bitcoin meet-up group and at an Internet of Human Things meet-up. These audiences had similar social profiles of the techno-elite within the dark net environment. While presenting evolving findings at these meetings, we clarified expressions and idiosyncratic language used by interview participants, explored the logics and continuities the interviewees presented, and gained technical knowledge of the cryptomarket environment. For example, in one meeting, our understandings of encrypted currencies and our reliance on a specific source were both critiqued, leading us to refine our arguments. Additionally, these member-checking exercises allowed us to present early findings from the research as a way of contributing back to the community. These presentations were then broadcast through multi-media formats (YouTube), which enabled members of the dark net market communities to access our findings in a low-risk and non-confrontational manner.

When curating and analysing the data, we focused on maintaining participant anonymity within our thematic alignments and in how we reported the findings. ${ }^{2}$ Even at this final stage of research practice, qualitative researchers working with sensitive topics face ethical and legal challenges, especially in areas where research participants may be asked to describe illegal activities. During the writing up of two articles for publication (Barratt et al., 2016; Maddox et al., 2016), we took extra precautions to remove potentially identifying information from the published interview quotations. For example, one interviewee described being arrested for drug supply charges related to Silk Road, and used a term to describe his sentencing that may have placed him in a

\footnotetext{
${ }^{2}$ Note that we have not outlined our analytic processes here. A summary of these can be found elsewhere (Maddox et al., 2016; Barratt et al., 2016).
} 
specific locality. In combination with public records of Silk Road related supply charges that we believe could have been linked to him, we remove this term from our paper. Similarly, we removed another participant's description of a personal tragedy that happened to him and impacted upon his drug use (relevant to one of the paper's research questions) that may have been identifying to people who knew him in combination with his age range.

\section{Ethical and methodological considerations across the workflow}

In this section, we describe considerations that interlocked across the stages of the research and informed ethnographic knowledge production more holistically. These considerations include the volatility of the field site as it relates to the enactment of a multi-sited study; researcher safety and positioning; the establishment and maintenance of data security; and the specific ethical-legal challenges we addressed in order to engage with a community involved in illicit practices.

The seizure of the Silk Road marketplace and forum challenged the assumptions raised in both Stage 1 and Stage 2 of the workflow that ethnographic fieldwork occurs in a singular defined location and that an online community can be identified through this. This idea of single-site social phenomena has long been contested, yet the first wave of online ethnographies were often restricted to singular online contexts like a specific forum or website (see Hine, 2008). Ethnographers have increasingly recognized that social life is dynamic and that objects of inquiry, such as people, things, information and ideas, are networked and dispersed across time and space (Falzon, 2009; Hine, 2008). Multi-sited ethnography, the frame we adopted in light of the disappearance of our field site, involves tracing networks or flows instead of constructing location-based boundaries around online communities.

Through our monitoring of the environment during the entry phase of Stage 2, it appeared that the established Silk Road community retained a semblance of shared identity, despite the closure of the marketplace. This cohesiveness was evident through the construction of a sub-forum on an alternative cryptomarket (Black Market Reloaded) specifically for Silk Road 'refugees', as well as their collective grieving and discussion within a specific Sub-Reddit ${ }^{3}$ dedicated to the Silk Road marketplace (see also Martin, 2014). From our ongoing digital presence, we identified previous Silk Road users interacting on (1) the forums of existing and new cryptomarkets, (2) Twitter, and (3) Reddit. Through monitoring of these locations, we also followed dispersed community members to a new dark net forum called The Hub and an

\footnotetext{
${ }^{3}$ Reddit is now one of the largest online communities. It originated as a community-driven platform for submitting, commenting and rating web content, but has increasingly focused on its own user-generated content (Singer et al., 2014).
} 
associated IRC ${ }^{4}$ channel. Through this approach we discovered that while the Silk Road ethos and community was unique in the dark net, once established, it continued to exist beyond the parameters of a single online location.

An unexpected but useful by-product of our research site disappearing just after our first interview was that we were able to reframe our project as retrospective, thereby reducing a perceived risk of participation in the project. We amended our interview materials to refer to Silk Road experiences in past tense and we added a question about the participant's response to the site shutdown. Paechter (2013) and Melrose (2002), researching traumatic experiences of divorce and sexual exploitation respectively, both argue that retrospective methodology in sensitive research can be more acceptable to participants who may have attained some degree of distance from these events. In our case, the sensitivity related to the potentially incriminating nature of admissions of involvement in drug markets, which has been previously described by online drug forum users as less risky to discuss in past tense (Barratt, 2011).

\section{Research safety in digital environments}

The social tensions and techno-legal disruptions common within these volatile spaces often evoke suspicion and insecurities, which can fuel trolling behaviours. Buckles et al. (2014) suggest that the personality profile of online trolls is characterised by a dark tetrad of sadism, psychopathy, and Machiavellianism. Their findings suggest that of all personality measures, sadism showed the most robust associations with trolling behaviour; from which they argue that cyber-trolling appears to be an Internet manifestation of everyday sadism. In line with this finding, the engagement of the troll with the recruitment post began with an attack on the veracity and risk implications of the research and then moved quickly to threats, ending in graphic death threats to the researcher, directed at A.M. In response to this, A.M contacted the forum moderators, who took action within the community to indicate that this behaviour was not acceptable and reiterated that they valued and supported the research. We cannot know the extent of risk to A.M.'s physical safety from this threat, however a lack of action on this threat suggests it was an act of everyday sadism. In response to this situation, we allowed the recruitment thread to 'drop' by not actively responding and moved the recruitment practice to other channels. Engaging across multiple channels could be considered sound research practice when working with contentious topics and stigmatised online communities where anonymity, flame wars and trolling are embedded in the culture.

While there has been some recent attention directed towards issues of researcher safety during fieldwork in the academic literature (Sang et al., 2012; Bahn, 2012), we were unaware of any discussions of violent threats to safety arising from digital

\footnotetext{
${ }^{4}$ IRC or Internet Relay Chat is a synchronous (real-time) communication channel (Reid, 1993) that has been operating for over 2 decades.
} 
ethnography, until very recently. Décary-Hétu and Aldridge (2015) describe an incident where one of them became aware of a threat to their life, due to an unfounded fear that the researchers were attempting to discover the location of an illicit marketplace's server when evidence of their web crawling was discussed with the marketplace's administrator. The incidents described here and by Décary-Hétu and Aldridge remind us that the safety of researchers working in digital spaces needs to be properly considered and safeguarded with the same care as is applied to conventional research engagements.

An additional researcher safety consideration related to us both identifying as female researchers in an almost all-male environment. As other female ethnographers have noted (e.g., Perrone, 2010; Poulton, 2012), this positioning affords both challenges and opportunities. The sites of research in our case include materials that objectify women as primarily for the sexual pleasure of men, and within these contexts, we were occasionally subject to the same positioning. For example, in the Silk Road community forum, M.B's contribution to a mainstream media article was commended (" 'Silk Road should actually prompt us to reconsider prohibition in its totality' ;) Love you Monica...X") but the discussion then progressed into jokes of a sexual nature. For A.M., when she entered the Hub IRC channel and described the research, she was asked "can i see your whispering eye?" (slang term for vagina) as well as "also, i'll do the interview". After A.M. responded with "v funny but not worth me opening up the interview space for thanks", further discussion included a claim that the interview offer was legitimate, a defence of the character of the potential interviewee ("[anonymized] is a good guy, he was just joking before") and the comment "this is just about the worst place to be a female honestly". A.M. continued to engage in this space although she did state "I don't mind talking about this stuff for research, but I'm not here to be harassed because I'm a chick hey'. Presenting as female in a mostly-male environment was not a choice, given the transparency of our real world identities in response to the security concerns of the community. While this meant that we had to negotiate these kinds of interactions, it also meant that our presence was somewhat novel, possibly drawing the attention and interest of participants to engage with us. We observe that the transparent and congruent conduct of ethnography in digital space for research purposes does not neutralise or negate the negotiation of gender identities and sexualisation, despite the apparent lack of embodied interactions.

Data security challenges were present before, during and after fieldwork. During the project lead-in time (Stage 1), we developed security protocols to ensure participant anonymity in all digital communications and the privacy of the interview transcripts from external viewers. To this end, during Stage 2, emails with participants were encrypted and all email correspondence was deleted from the university server, with anonymized records kept. Identifying data provided during the interview, such as the participant's pseudonym, avatar and disclosures, were removed from interview transcripts before they were saved. Furthermore, participant risks were minimised through keeping email correspondence solely to setting up interviews and ensuring that discussions conducted through IM focused on buyer (not vendor) experiences and 
avoided revealing personal data. These practices were aimed at garnering support from community members by meeting their expectations of sound security practices. We knew from our fieldwork that people who did not demonstrate technical competence relating to security were dismissed and ridiculed. Getting to know the community through participant observation (or 'listening', see Crawford, 2009) during Stage 1 ensured we understood the importance of utilising encryption and other security related practices. Indeed, upon entering the field, it was the first test to be passed before attaining some level of acceptance: for example, the Hub moderator in casual chat checked if A.M. had OTR through manually instigating it and then stating "oh you do have OTR". We believe that A.M.'s acceptance by the majority of the Hub community, despite her outsider status, would not have been possible without her practicing this level of technical expertise.

In addition to reducing personal vulnerability to attack, we refrained from using cloud-based data services where the servers were located in external countries (e.g. DropBox) to reduce risk of data breaching and snooping. Our team came from three different Australian universities and were located in different cities, so it was not possible to only use local servers. CloudStor+, a service run by Australia's Academic and Research Network (AARNet), uses local servers and is run specifically for the academic community to facilitate secure data sharing between authorized collaborators. While our main data security strategy was to anonymize all data immediately prior to saving, the use of CloudStor+ as a portal and back-up ensured our data remained secure and within our local jurisdiction.

\section{Ethical-legal challenges}

In some jurisdictions such as the United States, certificates of confidentiality may be obtained by researchers which protects them from having to reveal identifiable research information to authorities (Anderson and DuBois, 2007). In other jurisdictions including Australia where we are located, research data can theoretically be subpoenaed for use in law enforcement investigations of serious crimes (Loxley et al., 1997; Clough and Conigrave, 2008), although there are no local examples of subpoenaed researchers to our knowledge. However, in the UK, cultural geographer Bradley Garrett was arrested and charged with conspiracy to commit criminal damage after he publicly participated in 'place-hacking' or exploring hidden parts of London (Garrett, 2014). His research materials were seized and used to build the case against him and his 'urban explorers'. Even though his research had passed ethical review, the data he had collected under conditions of research confidentiality were used by authorities to prosecute him and 8 others (Garrett, 2014).

Within this legal context, we were also acutely aware of the highly interesting nature of our data for law enforcement, given that during the fieldwork period, there were ongoing criminal investigations of participants in the Silk Road community. Our approach was to ensure that our dataset would not contain specific information of use to law enforcement if it were subpoenaed, and this concern permeated all stages of the research. As previously outlined, our security procedures were designed to maintain participant anonymity through the use of encrypted communications, removal of 
identifying information prior to saving transcripts, and substitution of participant identification numbers in saved correspondence. Our study design was also affected by ethical concerns: we did not ask specifically about drug sales, although some respondents did describe their role as brokers in drug distribution. When this happened, we did not probe for more information. In addition to these practices, we asked about demographic information in broad categories to avoid collecting information that could be used to profile our participants. We also included clauses in our participant information sheet that noted the limits of confidentiality ('Please note that if specific information is supplied on activities dangerous to the public, the researchers would be obliged to notify appropriate authorities') and discouraged participants from providing any specific information about a crime or about their identity or that of other people (no-one actually did this).

Given the long lead-time we required to develop a working understanding of the Silk Road and related field-sites, it is unlikely that university ethics committees will have enough knowledge of the unique digital field-site we were working within (the dark net) to fully assess the potential of this research for harm. Although we informed our committee of changes in protocol, resulting in multiple ethics amendments, the onus ultimately fell back to us to make ethical decisions on an active, case-by-case basis. This project strongly demonstrates the contingent and dynamic nature of ethical conduct in research, as described eloquently by Markham (2005: 796):

In a very real sense, every method decision is an ethics decision, in that these decisions have consequences for not just research design but also the identity of the participants, the outcomes of our studies, and the character of knowledge which inevitably grows from our work in the field.

\section{An argument for active engagement}

In this paper we have outlined the three stages of our actively-engaged digital ethnography: (1) preparation; (2) field entry, engagement and exit; (3) production and dissemination. Specific challenges we described which cut across the workflow stages related to the volatility of the digital environment we were working within, the technical characteristics needed to successfully conduct our fieldwork, and the traditional concerns of research ethics, notably dealing with anonymity, consent, harm and overt/covert activities. Having described these challenges, nevertheless, we argue that engagement with dark net market participants through multi-sited digital ethnography offers some advantages when considered alongside more-common analyses of archival digital traces or Big Data studies, generally conducted without overt interaction with the communities under study. Our argument applies not just to dark net ethnography, but more broadly to digital interaction between researchers and people who engage in illicit or stigmatised practices. These advantages arise from both the longitudinal engagement and the interactive or participatory nature of our study.

As we have outlined in this paper, our approach involved tracing flows in a fracturing 
digital environment, and through this practice, we were able to be nimble and respond to cataclysmic events such as the loss of the research site. The practice of tracing cryptomarket participants through digital spaces as change was occurring gave us a sense of the volatility and dynamism of this environment, which is something that cannot be captured through webometric analyses involving scraping data at one time point (Aldridge and Décary-Hétu, 2014), although such change may be traced by repeated scrapings over an extended period (Soska and Christin, 2015).

Without active participation, it is impossible to recruit interviewees directly from the environment. Without conducting interviews, some of the research questions that interest us may be difficult or impossible to answer (for example, we were interested in an assessment of the differential impact of cryptomarket use on drug use and harm trajectories on Silk Road drug buyers). Participation also allows the practice of member checking nascent ideas, as in when we attended meet-ups of homologous communities to present early findings. While being alerted to some inaccuracies in the data interpretation, through this practice, we were able to confirm some commonalities between shared community experiences. Participating in the environment also enabled us to refine our interview methodology to one that would be more likely to be acceptable to the community. For example, by matching our technical protocols with community endorsed options, we demonstrated that we were abreast of security trends and community responses. This sensitivity helped us to establish legitimacy as researchers and build rapport. Finally, although active community participation involved a deft negotiation of competing discourses (and therefore took much time and energy), it was through these actions that we gained greater understanding of key community issues, like the tensions between publicity and secrecy. The live intersection between us and community members often required that we translate their cultural logics into our own, in an iterative fashion.

A further reason to conduct active participatory digital research with stigmatised populations is an ethical one. Hidden populations, especially those engaged in stigmatised activities, may be empowered by the opening of research channels to 'talk back' to researchers (Bakardjieva and Feenberg, 2001). The digital setting tends to reduce the researcher's power and control over the researcher-participant interaction, encouraging negotiation of meaning and dialogue (Barratt and Lenton, 2010; Barratt et al., 2015). As described in this paper, our experiences have demonstrated that active engagement through digital ethnography with hidden populations online forms an integral complement to digital trace analyses, for both methodological and ethical reasons. Active engagement adds richness, context and an opportunity for deliberate research participation by members of the community of interest, with which we can better interpret the findings of studies based solely on the analyses of their digital traces.

After presenting this research, conference delegates commented that we were 'brave to do this research' and that 'I would never do this work'. These comments may indicate a sense of fear around interacting with people who participate in illegal activities, and identifying ourselves as researchers within 'hacker' cultures, or just the inherent difficulties of working in this dynamic fieldwork space. We hope that this 
paper will assist other researchers who wish to enter similar spaces to consider the utility of a digital ethnographic approach and the pitfalls and opportunities of each ethical-methodological decision that occurs across the research process.

\section{Acknowledgements}

We thank Simon Lenton and Matthew Allen for their collaboration and stewardship on this project. We express heartfelt gratitude to the 17 Silk Road participants who shared their stories with us, and to the various community members who engaged with us and supported our work. We thank The Hub and the Dark Net Market Sub-Reddit for facilitating our work. We welcome your continued feedback on our ongoing work in this space. Earlier versions of this paper were presented at the Australian Consortium of Social and Political Research Inc. (ACSPRI) Social Science Methodology Conference 2014, Sydney, and the $6^{\text {th }}$ International Illicit Networks Workshop, Adelaide.

\section{Declaration of Conflicting Interests}

We declare that there are no conflicts of interests.

\section{Funding}

This research received no specific grant from any funding agency in the public, commercial, or not-for-profit sectors. The project was supported by internal funding from the National Drug Research Institute. The National Drug Research Institute in the Faculty of Health Sciences at Curtin University and the National Drug and Alcohol Research Centre at UNSW Australia are supported by funding from the Australian Government under the Substance Misuse Prevention and Service Improvement Grants Fund. Analysis and write-up by MB was facilitated via a National Health \& Medical Research Council Early Career Researcher Fellowship (APP1070140), administered through UNSW Australia. M.B. gratefully acknowledges the contribution to this work of the Victorian Operational Infrastructure Support Program received by the Burnet Institute. The funders played no further part in the research process, and the views expressed in this paper should not be seen as representative of the views of the funders. 


\section{References}

Ackland R. (2013) Web Social Science : Concepts, Data and Tools for Social Scientists in the Digital Age, Los Angeles: SAGE Publications.

Adler P. (1990) Ethnographic research on hidden populations: Penetrating the drug world. In: Lambert EY (ed) The collection and interpretation of data from hidden populations (NIDA Research Monograph No. 98). Rockville, MD: National Institute on Drug Abuse, 96-112.

Agar MH. (2011) Making sense of one other for another: Ethnography as translation. Language and Communication 31: 38-47.

Aldridge J and Décary-Hétu D. (2014) Not an 'Ebay for Drugs': The cryptomarket 'Silk Road' as a paradigm shifting criminal innovation. SSRN:

http://ssrn.com/abstract=2436643.

Anderson EE and DuBois JM. (2007) The need for evidence-based research ethics: A review of the substance abuse literature. Drug and Alcohol Dependence 86(2-3): 95105.

Ayling R and Mewse AJ. (2009) Evaluating Internet interviews with gay men. Qualitative Health Research 19: 566-576.

Bahn S. (2012) Keeping academic field researchers safe: Ethical safeguards. Journal of Academic Ethics 10(2): 83-91.

Bakardjieva M and Feenberg A. (2001) Involving the virtual subject. Ethics and Information Technology 2: 233-240.

Barratt MJ. (2011) Discussing illicit drugs in public internet forums: Visibility, stigma, and pseudonymity. In: Kjeldskov J and Paay J (eds) $C \& T$ '11. Proceedings of the Fifth International Conference on Communities and Technologies, Brisbane, Australia. New York, NY: ACM, 159-168.

Barratt MJ. (2012a) The efficacy of interviewing young drug users through online chat. Drug and Alcohol Review 31: 566-572.

Barratt MJ. (2012b) Silk Road: eBay for drugs [letter to the editor]. Addiction 107: 683.

Barratt MJ, Ferris JA and Winstock AR. (2014) Use of Silk Road, the online drug marketplace, in the UK, Australia and the USA. Addiction 109(5): 774-783.

Barratt MJ and Lenton S. (2010) Beyond recruitment? Participatory online research with people who use drugs. International Journal of Internet Research Ethics 3: 6986.

Barratt MJ, Lenton S and Allen M. (2013) Internet content regulation, public drug 
websites and the growth in hidden Internet services. Drugs: Education, Prevention, and Policy 20: 195-202.

Barratt MJ, Maddox A, Lenton S, et al. (2016) 'What if you live on top of a bakery and you like cakes?' - Exploring the drug use and harm trajectories before, during and after the emergence of Silk Road. International Journal of Drug Policy. Published online before print April 16, 2016, doi: 10.1016/j.drugpo.2016.04.006

Barratt MJ, Potter GR, Wouters M, et al. (2015) Lessons from conducting transnational Internet-mediated participatory research with hidden populations of cannabis cultivators. International Journal of Drug Policy 26: 238-249.

Borisov N, Goldberg I and Brewer E. (2004) Off-the-Record Communication, or, Why Not To Use PGP. Workshop on Privacy in the Electronic Society. Washington, D.C.

boyd d and Crawford K. (2012) Critical questions for big data. Information, Communication and Society 15(5): 662-679.

Bruns A. (2013) Faster than the speed of print: Reconciling 'big data' social media analysis and academic scholarship. First Monday 18(10).

Buckels EE, Trapnell PD and Paulhus DL. (2014) Trolls just want to have fun. Personality and Individual Differences 67: 97-102.

Clough A and Conigrave K. (2008) Managing confidentiality in illicit drugs research: Ethical and legal lessons from studies in remote Aboriginal communities. Internal Medicine Journal 38(1): 60-63.

Coomber R. (2011) Using the internet for qualitative research on drug users and drug markets: The pros, the cons and the progress. In: Fountain J, Frank VA and Korf DJ (eds) Markets, methods and messages. Dynamics in European drug research. Lengerich: Pabst Science Publishers, 85-103.

Crawford K. (2009) Following you: Disciplines of listening in social media. Continuum: Journal of Media and Cultural Studies 23(4): 525-535.

Creswell JW and Miller DL. (2000) Determining validity in qualitative inquiry. Theory Into Practice 39(3): 124-130.

Crowley JE. (2007) Friend or foe?: Self-expansion, stigmatized groups, and the researcher-participant relationship. Journal Of Contemporary Ethnography 36(6): 603-630.

Davis M, Bolding G, Hart G, et al. (2004) Reflecting on the experience of interviewing online: Perspectives from the Internet and HIV study in London. AIDS Care 16: 944-952.

Décary-Hétu D and Aldridge J. (2015) Sifting through the net: Monitoring of online 
offenders by researchers. European Research on Organised Crime 2: 122-141.

Dingledine R, Mathewson N and Syverson P. (2004) Tor: the second-generation onion router. SSYM'04 Proceedings of the 13th conference on USENIX Security Symposium. San Diego, CA, USA.

Ekbia H, Mattioli M, Kouper I, et al. (2015) Big data, bigger dilemmas: A critical review. Journal of the Association for Information Science and Technology 66(8): 1523-1545.

Ess C. (2009) Digital media ethics, Cambridge, England: Polity.

Falzon M-A. (2009) Introduction. In: Falzon M-A (ed) Multi-sited ethnography: Theory, praxis and locality in contemporary research. Surrey, England: Ashgate, 123.

Garrett B. (2014) Place-hacker Bradley Garrett: research at the edge of the law. Times Higher Education. Retrieved from

https://www.timeshighereducation.com/features/place-hacker-bradley-garrettresearch-at-the-edge-of-the-law/2013717.article

Gehl RW. (2014) Power/freedom on the dark web: A digital ethnography of the Dark Web Social Network. New Media \& Society. Published online before print October 15, 2014, doi: $10.1177 / 1461444814554900$

Hine C. (2008) Virtual ethnography: Modes, varieties, affordances. In: Fielding N, Lee RM and Blank G (eds) The SAGE handbook of online research methods. London, England: Sage, 257-270.

King SA. (1994) Analysis of electronic support groups for recovering addicts. Interpersonal Computing and Technology 2(3): 47-56.

Knox C. (2001) Establishing research legitimacy in the contested political ground of contemporary Northern Ireland. Qualitative Research 1(2): 205-222.

Koumartzis N and Veglis A. (2011) Internet regulation: The need for more transparent Internet filtering systems and improved measurement of public opinion on Internet filtering. First Monday 16(10).

Lee RM. (1993) Doing research on sensitive topics, London: Sage.

Lee RM. (1994) Dangerous fieldwork, London: Sage.

Loxley W, Hawks D and Bevan J. (1997) Protecting the interests of participants in research into illicit drug use: Two case studies. Addiction 92: 1081-1086.

Lupton D. (2015) Digital sociology, London: Routledge.

Maddox A, Barratt MJ, Allen M, et al. (2016) Constructive activism in the dark web: 
Cryptomarkets and illicit drugs in the digital 'demimonde'. Information, Communication and Society 19: 111-126.

Marcus GE. (1995) Ethnography in/of the world system: The emergence of multi-sited ethnography. Annual Review of Anthropology 24: 95-117.

Markham AN. (2005) The methods, politics, and ethics of representation in online ethnography. In: Denzin NK and Lincoln VS (eds) Handbook of qualitative research. 3rd ed. London: Sage, 793-820.

Martin J. (2014) Drugs on the dark net: How cryptomarkets are transforming the global trade in illicit drugs, New York, NY: Palgrave Pivot.

McCormack M, Adams A and Anderson E. (2013) Taking to the streets: The benefits of spontaneous methodological innovation in participant recruitment. Qualitative Research 13(2): 228-241.

McKee HA and Porter JE. (2009) The ethics of Internet research: A rhetorical, casebased process, New York, NY: Peter Lang.

McKenna KYA and Bargh JA. (1998) Coming out in the age of the Internet: Identity demarginalisation through virtual group participation. Journal of Personality and Social Psychology 75: 681-694.

Melrose M. (2002) Labour pains: Some considerations on the difficulties of researching juvenile prostitution. International Journal of Social Research Methodology 5(4): 333-351.

Miller PG and Sønderlund AL. (2010) Using the internet to research hidden populations of illicit drug users: A review. Addiction 105: 1557-1567.

Northcote J and Moore D. (2010) Understanding contexts: Methods and analysis in ethnographic research on drugs. In: Miller PG, Strang J and Miller PM (eds) Addiction research methods. Chichester, England: Wiley-Blackwell, 287-298.

Paechter C. (2013) Researching sensitive issues online: Implications of a hybrid insider/outsider position in a retrospective ethnographic study. Qualitative Research 13(1): 71-86.

Perrone D. (2010) Gender and sexuality in the field: A female ethnographer's experience researching drug use in dance clubs. Substance Use and Misuse 45: 717735.

Pew Research Center. (2014) Digital Life in 2025. Washington D.C.: Pew Research Center.

Pink S, Horst H, Postill J, et al. (2015) Digital ethnography: Principles and practice, London: Sage. 
Poulton E. (2012) 'If you had balls, you'd be one of us!' Doing gendered research: methodological reflections on being a female academic researcher in the hypermasculine subculture of 'football hooliganism'. Sociological Research Online 17(4): Article 4.

Rankin K. (2014) Tails above the Rest, Part II. Linux Journal April: 44-49.

Raynes-Goldie K. (2010) Aliases, creeping, and wall cleaning: Understanding privacy in the age of Facebook. First Monday 15(1).

Reid E. (1993) Electronic Chat: Social Issues on Internet Relay Chat. Media Information Australia 67: 62-70.

Singer P, Flöck F, Meinhart C, et al. (2014) Evolution of Reddit: From the front page of the internet to a self-referential community? Proceedings of the companion publication of the 23rd international conference on World wide web companion.

Seoul, Korea: International World Wide Web Conferences Steering Committee, 517522.

Soska K and Christin N. (2015) Measuring the longitudinal evolution of the online anonymous marketplace ecosystem. the 24th USENIX Security Symposium (USENIX Security'15). Washington, DC, 33-48.

Tanis M. (2008) What makes the internet a place to seek social support? In: Konijn EA, Utz S, Tanis M, et al. (eds) Mediated interpersonal communication. New York, NY: Routledge, 290-308.

van Buskirk J, Roxburgh A, Farrell M, et al. (2014) The closure of the Silk Road: what has this meant for online drug trading? Addiction 109(4): 517-518.

van Hout MC and Bingham T. (2013a) 'Silk Road', the virtual drug marketplace: A single case study of user experiences. International Journal of Drug Policy 24: 385391.

van Hout MC and Bingham T. (2013b) 'Surfing the Silk Road': A study of users' experiences. International Journal of Drug Policy 24: 524-529.

van Hout MC and Bingham T. (2014) Responsible vendors, intelligent consumers: Silk Road, the online revolution in drug trading. International Journal of Drug Policy 25: $183-189$.

Whiteman N. (2012) Undoing ethics. Rethinking practice in online research. New York: Springer.

Williams M. (2007) Policing and cybersociety: The maturation of regulation within an online community. Policing and Society 17(1): 59-82.

Yassour-Borochowitz D. (2012) "Only if she is sexy". Equality, Diversity and Inclusion: An International Journal 31(5/6): 402-417. 